\title{
Recommendation of RILEM TC 237-SIB on complex Poisson's ratio characterization of bituminous mixtures
}

\author{
A. Graziani 1 - H. Di Benedetto $\cdot$ D. Perraton $\cdot$ C. Sauzéat • \\ B. Hofko $\cdot$ L. D. Poulikakos $\cdot$ S. Pouget
}

Received: 1 December 2016/Accepted: 30 January 2017 / Published online: 27 February 2017

(C) RILEM 2017

\begin{abstract}
This recommendation describes how to characterize the isotropic three-dimensional linear viscoelastic behaviour of bituminous mixtures through the simultaneous measurement of the complex Young's modulus $\left(E^{*}\right)$ and the complex Poisson's ratio $\left(v^{*}\right)$. The guidelines given herein are based on the results of a RILEM round robin test organized by the RILEM Technical Committee 237-SIB “Testing and characterization of sustainable innovative bituminous

This recommendation was developed by the task group TG3 within RILEM TC 237-SIB consisting of Andrea Graziani, Hervé Di Benedetto, Daniel Perraton, Cédric Sauzéat, Bernhard Hofko, Lily D. Poulikakos, Simon Pouget. The draft recommendation was submitted for approval to the full TC and subsequently approved by RILEM TC 237-SIB.
\end{abstract}

TC Chairman: Manfred N. Partl (EMPA - Swiss Federal Laboratories for Materials Science and Technology, Dubendorf, Switzerland).

TC Secretary: Emmanuel Chailleux (IFSTTAR - French institute of science and technology for transport, development and networks, Nantes, France).

TG3 Group Leader: Hervé Di Benedetto (University of Lyon, ENTPE - LTDS (UMR CNRS 5513), Vaulx-en-Velin, France).

TC Members: G. Airey (UK), A. Apeagyei (UK), I. Artamendi (UK), H. Baaj (Canada), H.U. Bahia (USA), W. Bankowski (Poland), D. Broere (The Netherlands), Y. Brosseaud (France), W. G. Buttlar (USA), F. Canestrari (Italy), A. Chabot (France), E. Chailleux (France), E. Dave (USA), J. De Visscher materials and systems" and focus on the testing procedure, measurement setup, data analysis and presentation of results. Indications for the preparation of a tests report are also given.

Keywords Complex Young's modulus · Complex Poisson's ratio $\cdot$ Three-dimensional characterization · Linear viscoelasticity $\cdot$ Bituminous mixture

(Belgium), H. Di Benedetto (France), T. Gabet (France), M. Gajewski (Poland), V. Gaudefroy (France), G. Ferrotti (Italy), W. Grady (The Netherlands), A. Graziani (Italy), J. Grenfell (UK), F. Hammoum (France), B. Hofko (Austria), M. Hugener (Switzerland), I. Ishai (Israel), N. Kringos (Sweden), G.A. Leegwater (The Netherlands), E. Levenberg (Israel), A. Loizos (Greece), X. Lu (Sweden), P. Marsac (France), A. Millien (France), K. Mollenhauer (Germany), F. Morea (Argentina), V. Mouillet (France), P. Muraya (Norway), M.N. Partl (Switzerland), D. Perraton (Canada), C. Petit (France), K. Petros (USA), L. Porot (The Netherlands), Pouget S. (France), L. Poulikakos (Switzerland), C. Raab (Switzerland), S. Said (Sweden), C. Sangiorgi (Italy), C. Sauzéat (France), T. Scarpas (The Netherlands), H. Soenen (Belgium), D. Sybilsky (Poland), G. Tebaldi (Italy), A. Vanelstraete (Belgium), M. Wistuba (Germany), L. Zanzotto (Canada), A. Zofka (Poland).

A. Graziani $(\bowtie)$

Università Politecnica delle Marche, Ancona, Italy

e-mail: a.graziani@univpm.it

H. Di Benedetto · C. Sauzéat

ENTPE - LTDS (UMR CNRS 5513), University of Lyon,

Vaulx-en-Velin, France 


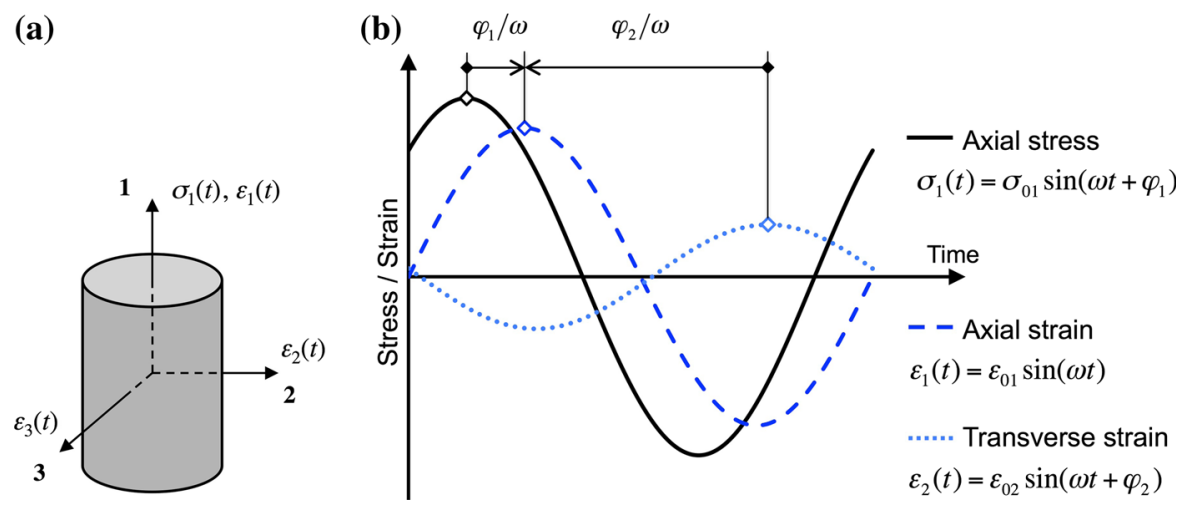

Fig. 1 Axial test on a cylindrical specimen: a identification of stress and strain principal directions; $\mathbf{b}$ time-domain representation of stress and strain sinusoidal signals

\section{Scope}

This recommendation gives guidance for the characterisation of the three-dimensional (3D) linear viscoelastic (LVE) behaviour of bituminous mixtures considered as isotropic. Specifically, it refers to the simultaneous measurement of the complex Young's modulus $\left(E^{*}\right)$ and the complex Poisson's ratio $\left(v^{*}\right)$ by means of sinusoidal axial tests on cylindrical specimens.

The recommendations are based on the results of a RILEM round robin test (RRRT) organized by the RILEM Technical Committee 237-SIB “Testing and characterization of sustainable innovative bituminous materials and systems". This document contains guidelines on testing procedure, measurement setup, data analysis and presentation of results. Additional details on the results of the RRRT may be found in Perraton et al. [1] and Graziani et al. [2].

D. Perraton

ÉTS, University of Québec, 1100, Notre-Dame Ouest, Montréal, QC, Canada

B. Hofko

Vienna University of Technology, Vienna, Austria

L. D. Poulikakos

EMPA, Swiss Federal Laboratories for Materials Science and Technology, Dübendorf, Switzerland

\section{S. Pouget}

Research and Development Department, EIFFAGE Infrastructures, Corbas Cedex, France

\section{Definition of complex Young's modulus and Poisson's ratio}

Let us consider a cylindrical specimen of an isotropic, linear viscoelastic (LVE) material subjected only to a steady-state sinusoidal loading in axial direction (Fig. 1a). Although the stress state is uniaxial $\left[\sigma_{1}(t) \neq 0, \sigma_{2}(t)=\sigma_{3}(t)=0\right]$, the response strain field is tree-dimensional and is characterized by two independent strains: $\varepsilon_{1}(t)$ and $\varepsilon_{2}(t)=\varepsilon_{3}(t)$. Hence, the steady-state stress-strain response of the material can be described by the following complex exponentials (or phasors):

$\sigma_{1}^{*}(\omega)=\sigma_{01} \exp \left[\mathrm{j}\left(\omega t+\varphi_{1}\right)\right]$

$\varepsilon_{1}^{*}(\omega)=\varepsilon_{01} \exp (\mathrm{j} \omega t)$

$\varepsilon_{2}^{*}(\omega)=\varepsilon_{02} \exp \left[\mathrm{j}\left(\omega t+\varphi_{2}\right)\right]$

where $\mathrm{j}$ is the imaginary unit, $\omega$ is the angular frequency, $\sigma_{01}$ is the stress amplitude, $\varepsilon_{01}$ and $\varepsilon_{02}$ are the amplitudes of axial and transverse strain, respectively, and $\varphi_{1}$ and $\varphi_{2}$ are phase angles. Since phase shifts between phasors actually represent time shifts between the corresponding time domain signals $\left(\varphi_{i}=\omega \Delta t_{i}\right), \varphi_{1}$ and $\varphi_{2}$ represent the time shifts of $\sigma_{1}(t)$ and $\varepsilon_{2}(t)$ with respect to $\varepsilon_{1}(t)$, which was customarily assumed as the reference signal (Fig. 1b).

The complex Young's modulus and the complex Poisson's ratio are defined as follows [3]:

$E^{*}(\omega)=\frac{\sigma_{1}^{*}(\omega)}{\varepsilon_{1}^{*}(\omega)}=\frac{\sigma_{01}}{\varepsilon_{01}} \exp \left(\mathrm{j} \varphi_{E}\right)$ 
Fig. 2 Complex plane representation of the sinusoidal steady state response of a LVE material: a stress and strain phasors; b complex response functions $E^{*}$ and $v^{*}$ (a)

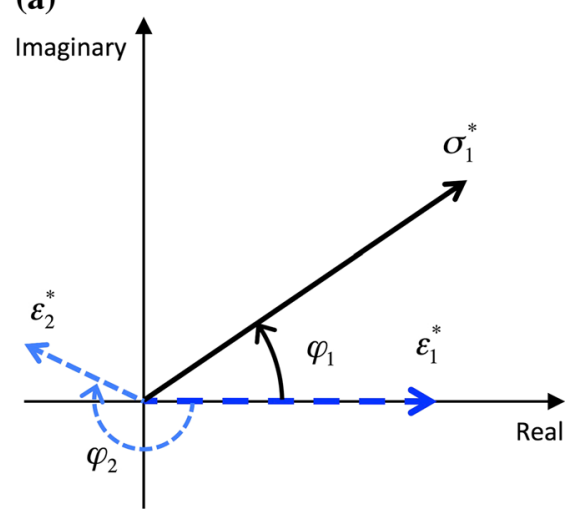

(b)

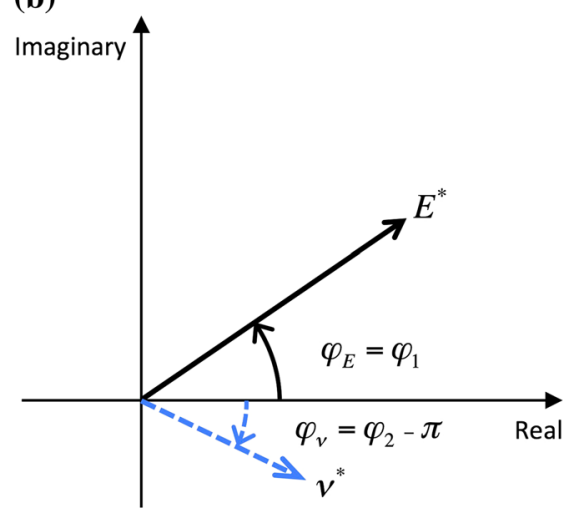

$v^{*}(\omega)=-\frac{\varepsilon_{2}^{*}(\omega)}{\varepsilon_{1}^{*}(\omega)}=\frac{\varepsilon_{02}}{\varepsilon_{01}} \exp \left(\mathrm{j} \varphi_{v}\right)$

where $\left|E^{*}\right|=E_{0}=\sigma_{01} / \varepsilon_{01},\left|v^{*}\right|=v_{0}=\varepsilon_{02} / \varepsilon_{01}$ are the norms (or absolute values) and $\varphi_{E}=\varphi_{1}, \varphi_{v}=$ $\varphi_{2}-\pi$ are the phase angles. The complex quantities described by Eqs. 1-5 are depicted in Fig. 2.

It is also possible to use the following notations:

$E^{*}(\omega)=E_{1}(\omega)+\mathrm{j} E_{2}(\omega)$

$v^{*}(\omega)=v_{1}(\omega)+\mathrm{j} v_{2}(\omega)$

where $E_{1}, E_{2}$ are the real and imaginary parts of the complex Young's modulus, also called storage modulus and loss modulus, respectively, whereas $v_{1}, v_{2}$ are the real and imaginary parts of the complex Poisson's ratio.

For an isotropic material, the following relations for the description of the 3D LVE behaviour are also valid:

$$
\begin{aligned}
K^{*} & =\frac{E^{*}}{3\left(1-2 v^{*}\right)} \\
G^{*} & =\frac{E^{*}}{2\left(1+v^{*}\right)}
\end{aligned}
$$

where $K^{*}$ is the complex bulk modulus and $G^{*}$ is the complex shear modulus. It has been remarked that the practical applications of Eqs. 8 and 9, requires that the source functions be determined simultaneously on the same specimen, in order to guarantee identical initial and boundary conditions [4].

\section{Simultaneous measurement of complex Young's modulus and complex Poisson's ratio}

It is recommended that the simultaneous measurement of $E^{*}$ and $v^{*}$ of bituminous mixtures is carried out by means of sinusoidal axial tests on cylindrical specimen. The general test setup should be based on those currently adopted for the measurement of $E^{*}[5,6]$. Measurement of the transverse (either diametral or circumferential) strain is also required in order to calculate $v^{*}$ and obtain a 3D isotropic characterization. Such a test configuration provides homogeneous stress and strain fields in the central part of the specimen, which allows the calculation of $v^{*}$ through the direct comparison of axial and transverse strains according to Eq. 5.

Although for bituminous mixtures the phase angle of $v^{*}$ is very small, generally less than $10^{\circ}$, its determination is highly recommended. Assuming $\varphi_{v}=0$ (i.e. assuming axial contraction/dilation is in counterphase with transverse contraction/dilation) may lead to inconsistencies in the description of the 3D response. For example, from Eqs. 8 and 9 it would follow that bituminous mixtures have the same loss angle in bulk and shear deformation modes. In addition Poisson's ratio value would be real and constant.

\subsection{Specimen preparation}

Cylindrical specimens should be prepared following accepted standards for complex Young's modulus testing. It is remarked that specimens prepared with 
different compaction equipment (e.g. gyratory compactor, slab compactor) or cored in different directions relative to the compaction direction, may show a different degree of anisotropy in the LVE response. Hence, it is recommended to clearly report the compaction method, the coring direction and the direction(s) used for the measurement of transverse strain.

\subsection{Small strain domain}

The simultaneous measurement of $E^{*}$ and $v^{*}$ of bituminous mixtures shall be carried out within the LVE domain. For bituminous mixtures this requires the application of "small" axial strain amplitudes, generally lower than $100 \times 10^{-6} \mathrm{~m} / \mathrm{m}$. It is remarked that such a linearity limit was established based solely on the measurement of $E^{*}$ [7-9] and therefore linearity tests should be performed in case of doubt.

It is worth noting that the selection of such a low value for the axial strain amplitude $\varepsilon_{01}$ has practical consequences on the transverse strain amplitude $\varepsilon_{02}$ occurring during the test. For example, if the Poisson's ratio norm is $v_{0}=0.2$, the transverse strain amplitude will be in the order of $10 \times 10^{-6} \mathrm{~m} / \mathrm{m}$. This should be considered when selecting the transverse strain measuring system.

\subsection{Testing modality}

It is recommended to apply a sinusoidal, i.e. tension/compression (TC) loading history. An haversine loading history, i.e. cyclic compression (CC) or cyclic tension, may also be applied. In the latter case, only the sinusoidal component of time-domain excitation and response functions shall be analysed to calculate both $E^{*}$ and $v^{*}[10]$.

Testing may be carried out either in strain or stress controlled mode. In the latter case the axial stress amplitude $\sigma_{01}$ shall be set to obtain the target steadystate strain amplitude $\varepsilon_{01}$.

It is to be underlined that due to rather low applied frequencies (less than about $10 \mathrm{~Hz}$ ) inertia effects are negligible. The considered tests are quasi-static cyclic tests and should not be called "dynamic tests" as sometimes stated. Dynamic tests considering back analysis from waves propagating in the specimen can also be used to obtain $E^{*}$ and $v^{*}[11-13]$.

\subsection{The steady-state}

The calculation of frequency-domain material LVE functions should be made during steady-state regime. From a practical point of view, a minimum number of loading cycles should be applied in order to allow the progressive fading away of transient effects due to initial rest conditions. Two to three cycles seems enough to obtain this regime [14]. On the other hand, the number of loading cycles should be limited, so that heating and damage due to repeated loading is limited $[9,15]$ and, for CC testing, hardening (or softening) due to creep deformation is negligible.

Generally the number of loading cycles applied should be selected with some judgment, considering test temperature and frequency. Moreover, evolution of the material response during the test should be carefully checked.

\subsection{Testing temperature and frequency}

Similar to complex Young's modulus testing, the range of testing temperature and frequency shall consider potentiality and limitations of available laboratory equipment.

Generally, the sinusoidal excitation is produced using a closed-loop control system, therefore it is recommended to check the quality of the sinusoidal excitation, either stress or strain, applied to the specimen. Hence, the data acquisition system should give access to the raw (unprocessed) values of stress and strain.

\subsection{Stress and strain measurement systems}

Measurement of axial stress and strain should be carried out following accepted standards for complex Young's modulus testing.

It is recommended to measure axial and transverse strain locally on the specimen. In particular, transverse strain should be measured at mid-height of the specimen using traditional glued strain gauges or non-contact sensors. It is remarked that in the first case an average circumferential strain is measured, whereas in the second case a localized diametral strain is detected. It is also recommended to use a unique data acquisition unit, in order to obtain perfectly synchronized data and avoid systematic 
errors in the measurement of phase lags. Given the small amplitude of the transverse strain (Sect. 3.2), high accuracy and precision of the strain measuring system is mandatory.

\subsection{Temperature control and measurement}

Similar to complex Young's modulus measurements, the use of a suitable thermal chamber to control the temperature of the specimen during the test is recommended. Temperature readings should be performed using probes installed on the specimen surface, e.g. platinum resistance thermometers (PT100) or K-type thermocouples. Temperature sensors should be adequately coated in order to avoid fluctuation due to air temperature variations.

When strain is measured using strain gauges, adequate temperature compensation circuits should be used. This normally requires the use a dummy specimen placed inside the thermal chamber.

\subsection{Calculation of $\boldsymbol{E}^{*}$ and $\boldsymbol{v}^{*}$}

The calculation of $E^{*}$ and $v^{*}$ should be carried out, for each testing temperature and frequency, through the direct application of Eqs. 4 and 5. It is recommended to determine the source parameters $\left(\sigma_{01}, \varepsilon_{01} \varepsilon_{02}\right.$, $\left.\varphi_{1}, \varphi_{2}\right)$ by performing a sinusoidal regression analysis of the measured discrete-time signals (stress and strains).

Due to the small value of the transverse strain and because of the small phase lag between axial and transverse strain, additional uncertainties due to the numerical regression procedure may have a significant impact on $v^{*}$ values. Hence it is recommended to use numerical algorithms based on linear regression theory, which provide closed form estimates of the source parameters, i.e. based only on measured data.

\subsection{Cole-Cole and Black diagrams for $\boldsymbol{v}^{*}$}

Similar to the current practice for $E^{*}$, Black and ColeCole diagrams are a very simple and practical way to plot and analyse $v^{*}$ values. Different from $E^{*}$, the absolute value of $v^{*}$ should be plotted on a linear scale. If $v^{*}$ values can be represented by a unique curve in the Black or Cole-Cole diagrams, the time-temperature superposition principle can be considered valid.

As an example, data measured within the RRRT on two different mixtures are reported in Fig. 3 (asphalt concrete with continuous grading curve) and Fig. 4 (asphalt concrete with gap-graded curve). Values measured at lower temperatures are situated in the areas indicated with "L", whereas values measured at higher temperatures gradually plot towards the areas indicated with "H". The plotted results clearly show that the upper limit of the norm of $v^{*}$ is not necessarily 0.5 , as commonly assumed.

\subsection{Master curves for $v^{*}$}

Master curves for $v^{*}$ components $\left(v_{0}, \varphi_{v}, v_{1}\right.$ and $\left.v_{2}\right)$ can be obtained following the same procedures normally adopted for $E^{*}$. Upon selection of a reference temperature the measured values at all other test temperatures are shifted along the log-frequency axis until a unique curve is obtained. If the time-
Fig. 3 Example complex Poisson's ratio measurements on asphalt concrete with continuous grading curve: a Black diagram; b Cole-Cole diagram. $L(H)$ indicates the domain with lower (higher) temperatures
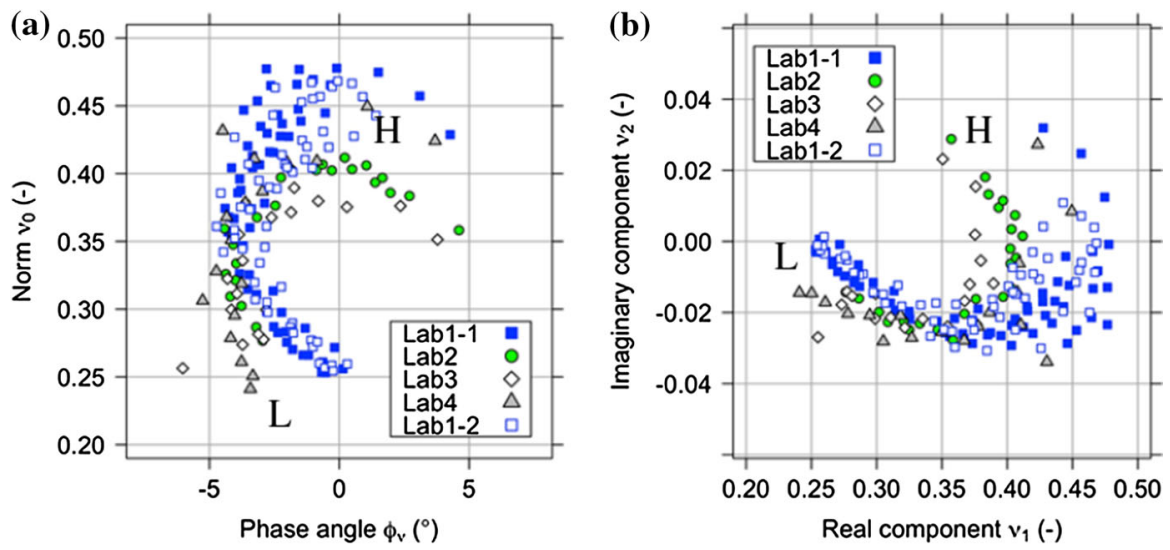
Fig. 4 Example complex Poisson's ratio measurements on asphalt concrete with gap-graded curve: a Black diagram; b Cole-Cole diagram. $L(H)$ indicates the domain with lower (higher) temperatures
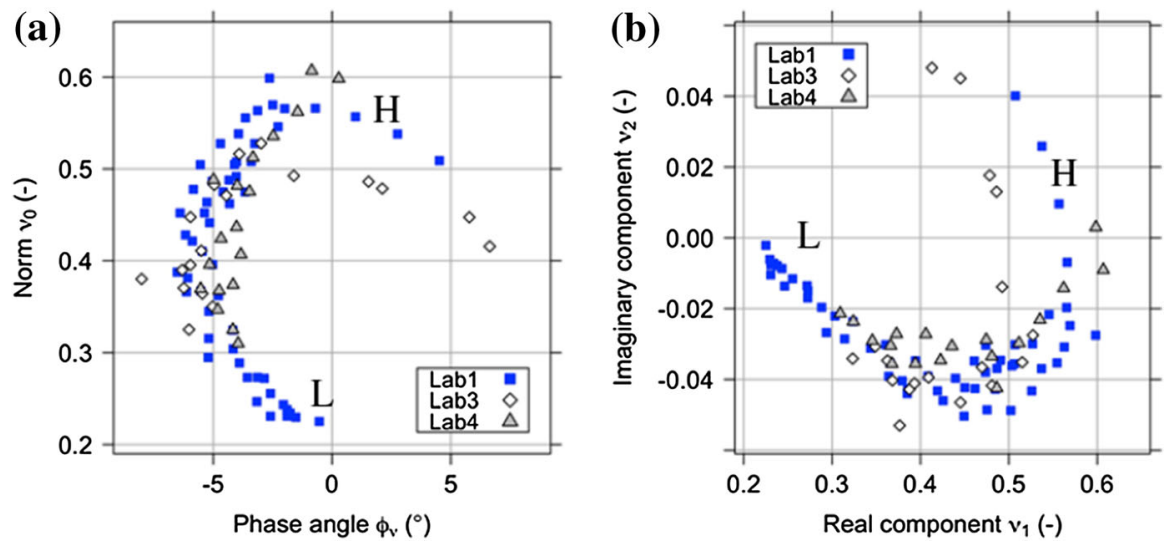

Fig. 5 Master curves of the components of complex Poisson's ratio: a norm; b phase angle

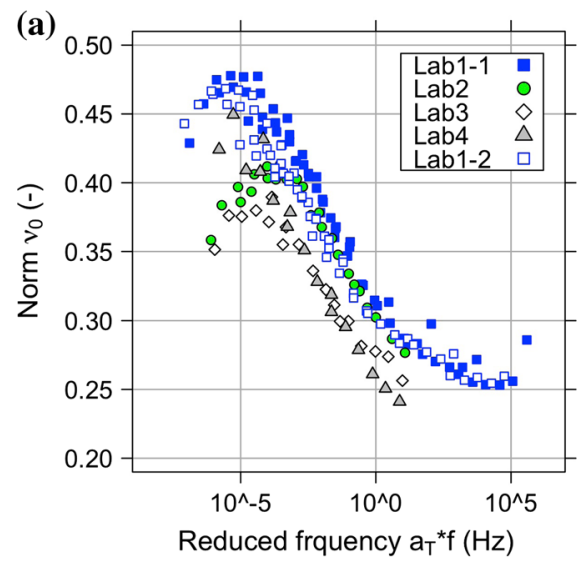

temperature superposition principle is valid, the same shift factors may be used for all $v^{*}$ components.

Example master curves of $v_{0}$ and $\varphi_{v}$ obtained within the RRRT are reported in Fig. 5. It can be noted that master curves of $v_{0}$ are not monotonic and that master curves of $\varphi_{v}$ show a change of sign. The same shift factors were obtained for $E^{*}$ and $v^{*}$ as already stated by various authors [16-19] .

\subsection{Precision of testing}

Results of the RRRT were not conclusive regarding repeatability and reproducibility of $E^{*}$ and $v^{*}$ values. However, the data plotted in Figs. 3, 4 and 5 may give an indication of the variability of $v^{*}$ measured according to the present recommendation. In the RRRT the chosen axial strain level was $50 \times 10^{-6}$ $\mathrm{m} / \mathrm{m}$, thus a variation of about 0.02 for the Poisson's ratio norm corresponded to a variation of $10^{-6} \mathrm{~m} / \mathrm{m}$ in the diametral or circumferential strain. For specimens with diameter of $100 \mathrm{~mm}$, this corresponded to a change in diameter or approximately $0.10 \times 10^{-3} \mathrm{~mm}$ or a change in circumference of about $0.31 \times 10^{-3}$ $\mathrm{mm}$. Clearly both the resolution and the precision of the measurement chain, as well as the accuracy of regression analysis are crucial in order to obtain good repeatability and reproducibility.

\section{Concluding remarks}

Sinusoidal axial tests on cylindrical specimens allow the simultaneous measurement of the complex Young's modulus $\left(E^{*}\right)$ and the complex Poisson's ratio $\left(v^{*}\right)$ and thus can be used to characterize the isotropic 3D LVE behaviour of bituminous mixtures.

According to the present recommendation, the test report should contain: 
- The relevant mixture and specimen details, e.g. mixture type, compaction method, specimen dimensions, coring direction;

- The description of the test setup, including at least: load measuring system, axial and transverse strain measuring system, data acquisition frequency;

- The values of the following testing variables: temperature, loading frequency, number of loading cycles (for each temperature and frequency), target axial strain amplitude;

- The numerical technique adopted to calculate the sinusoidal stress and strain amplitudes and phase angles (Eqs. 1-3);

- The calculated values of the complex material functions $E^{*}$ and $v^{*}$, expressed both in trigonometric form (Eqs. 4, 5) and algebraic form (Eqs. 6, 7) and the corresponding graphical representation in the Black and Cole-Cole diagrams;

- The graphical representation of norm and phase angle of $E^{*}$ and $v^{*}$ as a function of reduced frequency (master curves).

\section{Compliance with ethical standards}

Conflict of interest The authors declare that they have no conflict of interest.

\section{References}

1. Perraton D, Di Benedetto H, Sauzéat C, Hofko B, Graziani A, Nguyen QT, Pouget S, Poulikakos LD, Tapsoba N, Grenfell J (2016) 3Dim experimental investigation of linear viscoelastic properties of bituminous mixtures. Mater Struct. doi:10.1617/s11527-016-0827-3

2. Graziani A, Di Benedetto H, Perraton D, Sauzéat C et al (2016) Three-dimensional characterisation of linear viscoelastic properties of bituminous mixtures. RILEM State of the Art Report, Springer (in publication)

3. Di Benedetto H, Delaporte B, Sauzeat C (2007) Three dimensional linear behavior of bituminous materials: experiments and modeling. Int J Geomech 7:149-157. doi:10.1061/(ASCE)1532-3641(2007)7:2(149)

4. Tschoegl NW, Knauss WG, Emri I (2002) Poisson's ratio in linear viscoelasticity - a critical review. Mech Time Depend Mater 6(1):3-51

5. EN 12697-26 (2012) Bituminous mixtures-test methods for hot mix asphalt-Part 26: Stiffness. CEN, Comité Européen de Normalisation, Brussels

6. AASHTO T 342 (2009) Standard method of test for determining dynamic modulus of hot mix asphalt (HMA).
American Association of State Highway and Transportation Officials, Washington

7. Airey GD, Rahimzadeh B, Collop AC (2003) Viscoelastic linearity limits for bituminous materials. Mater Struct 36(10):643-647

8. Di Benedetto H, Partl MN, Francken L, Saint André CDLR (2001) Stiffness testing for bituminous mixtures. Mater Struct 34(2):66-70

9. Nguyen QT, Di Benedetto H, Sauzeat C, Tapsoba N (2013) Time temperature superposition principle validation for bituminous mixes in the linear and nonlinear domains. J Mater Civ Eng 25(9):1181-1188

10. Graziani A, Bocci M, Canestrari F (2014) Complex Poisson's ratio of bituminous mixtures: measurement and modeling. Mater Struct 47(7):1131-1148

11. Gudmarsson A, Ryden N, Di Benedetto H, Sauzéat C, Tapsoba N, Birgisson B (2014) Comparing linear viscoelastic properties of asphalt concrete measured by laboratory seismic and tension-compression tests. J Non-destr Eval 33(4):571-582

12. Gudmarsson A, Ryden N, Di Benedetto H, Sauzéat C (2015) Complex modulus and complex Poisson's ratio from cyclic and dynamic modal testing of asphalt concrete. Constr Build Mater 88:20-31

13. Mounier D, Di Benedetto H, Sauzeat C (2012) Determination of bituminous mixtures linear properties using ultrasonic wave propagation. Constr Build Mater 36:638-647. doi:10.1016/j.conbuildmat.2012.04.136

14. Gayte P, Di Benedetto H, Sauzéat C, Nguyen QT (2015) Influence of transient effects for analysis of complex modulus tests on bituminous mixtures. Road Mater Pavement Des. doi:10.1080/14680629.2015.1067246

15. Di Benedetto H, Nguyen QT, Sauzeat C (2011) Nonlinearity, heating, fatigue and thixotropy during cyclic loading of asphalt mixtures. Road Mater Pavement Des 12(1):129-158

16. Nguyen HM, Pouget S, Di Benedetto H, Sauzeat C (2009) Time-temperature superposition principle for bituminous mixtures. Eur J Environ Civ Eng 13(9):1095-1107. doi:10. 3166/EJECE.13

17. Pham NH, Sauzéat C, Di Benedetto H, Gonzalez-Leon JA, Barreto G, Nicolai A, Jakubowski M (2015) Reclaimed asphalt pavement and additives influence on 3D linear behaviour of warm mix asphalts. Road Mater Pavement Des 16(3):569-591. doi:10.1080/14680629.2015.1021108

18. Pouget S, Sauzéat C, Di Benedetto H, Olard F (2012) Modeling of viscous bituminous wearing course materials on orthotropic steel deck. Mater Struct 45(7):1115-1125. doi:10.1617/s11527-011-9820-z

19. Tiouajni S, Di Benedetto H, Sauzeat C, Pouget S (2011) Approximation of Linear Viscoelastic Model in the 3 Dimensional Case with Mechanical Analogues of Finite Size. Road Mater Pavement Des 12(4):897-930. doi:10. 3166/RMPD. 12 